\title{
Descobrindo definições matemáticas no contexto de investigação \\ histórica: o caso da sequência generalizada de Fibonacci
}

\section{Francisco Regis Vieira Alves}

Instituto Federal de Educação, Ciência e Tecnologia do Ceará (IFCE)

fregis@ifce.edu.br

\section{Resumo}

Nesta proposição de aula apresentamos uma abordagem de investigação no contexto da História da Matemática, relativamente a um assunto pouco abordado no âmbito da graduação, envolvendo a Sequência Generalizada de Fibonacci - SGF. Trazemos, pois, alguns artigos da década de 60 que ainda preservam um valor pedagógico, desde que sejam passíveis de adaptação/exploração, sobretudo, com arrimo da tecnologia, num contexto de um curso de licenciatura de Matemática.

Palavras-chave: Sequência Generalizada de Fibonacci - SGF. História da Matemática. Atividades de investigação.

\section{Discovering mathematical definitions in the context of historical research: the case of generalized Fibonacci sequence}

\begin{abstract}
In this class proposition we present a research approach in the context of the history of Mathematics, for a subject rarely adressed in the undergraduate context, involving the Generalized Fibonacci Sequence - GFS. We bring, therefore, some articles of the 60 decade that, still preserve a padagogical value, provided they are subject to changes/exploitation especially with the technology's help, in a context of a mathematics degree couse.
\end{abstract}

Keywords: Generalized Fibonacci Sequence. History of Mathematics. Research activities.

\section{Introdução}

Em alguns dos nossos trabalhos, temos abordado a possibilidade de estender a sequência emblemática de Fibonacci ao conjunto dos números inteiros. De modo específico, estruturamos uma atividade de sala de aula que descreve/indica uma abordagem para a discussão da "sequência extendida" definida por $\left(f_{n}\right)_{n \in Z}$, em vez de $\left(f_{n}\right)_{n \in I N}$ (ALVES; BORGES NETO, 2011; ALVES, 2013). Dois elementos devem ser destacados nesses trabalhos, a saber: (i) a influência de um olhar influenciado pela História da Matemática e (ii) o uso de uma abordagem metodológica de ensino com arrimo na tecnologia. Assinalamos, ainda, que uma concepção maior da atividade reside em 
inserir os alunos numa incursão envolvendo a produção/descrição de definições matemáticas formais e, neste contexto, estruturar situações didáticas que possibilitam a generalização de noções desconsideradas pelos autores de livros de História da Matemática. Para tanto, discutiremos outras sequências recursivas que possuem propriedades relacionadas com a noção de Sequência Generalizada de Fibonacci - SGF (KOSHY, 2011).

\section{Atividade Proposta}

Neste trabalho descrevemos uma atividade a ser realizada com alunos de Ensino Superior que cursam a disciplina de História da Matemática. O problema consiste em considerar os itens envolvendo duas definições que se inserem num contexto de investigação:

(I) Considerar a sequência definida pela seguinte relação $p_{n}=p_{n-1}+p_{n-2}+p_{n-3}, n \geq 3$ (WADDILL; SACKS, 1967, p. 210).

(II) Considerar a sequência dada por $q_{n}=q_{n-1}+q_{n-2}+q_{n-3}+\mathrm{q}_{n-4}, n \geq 4$ (WADDILL; SACKS, 1967, p. 210).

(III) Considerar os termos da sequência $(p, q, r, s, t, p+q+r+s+t, p+2 q+2 r+2 s+2 t, \ldots)$ e indicar uma fórmula de recorrência para a mesma.

\section{Questionamentos:}

1. Escolhendo os seguintes termos $p_{0}=0, p_{1}=p_{2}=1, \mathrm{p}_{3}=2, p_{4}=4$, podemos descrever os primeiros dez números desta sequência à direita $(n>0)$ e à esquerda $(n<0)$ ?

2. Que nome ou terminologia poderíamos atribuir às sequências numéricas indicadas logo acima por $\left\{p_{n}\right\}_{n \in Z}$ (I) e por $\left\{q_{n}\right\}_{n \in Z}$ (II) ?

3. Definindo a seguinte sequência $\left\{R_{\tilde{n}}\right\}_{n \in I N}$ de modo que $\left\{R_{0}=p_{1}-p_{0}\right.$ e $\left.R_{n}=p_{n-1}+p_{n-2}\right\}$, que relações/propriedades esta sequência possui com o item (I)? Explique!

4. Semelhantemente ao caso da sequência de Fibonacci, relacionada com o número de ouro, obtido como uma das raízes da função polinomial $x^{2}-x-1=0$, que equação polinomial relacionamos com a sequência definida em (I) e (II)?

\section{Discussão}

O objetivo desta atividade é explorar as possibilidades de adaptação, adequação e generalização de certas concepções dos estudantes, no contexto de investigação históricas, atinentes ao conceito Sequência Generalizada de Fibonacci - SGF. 


\section{Atividades previstas}

Com embasamento matemático, apoiado pelos conhecimento prévios acerca da sequência de Fibonacci o aluno poderá perceber que:

Item (I): os valores preliminares da sequência $p_{n}=p_{n-1}+p_{n-2}+p_{n-3}, n \geq 3$ podem ser $p_{0}=0, p_{1}=p_{2}=1 \mathrm{e} / \mathrm{ou} p_{0}=1, p_{1}=0, p_{2}=1$, As sequências assim obtidas podem ser indicadas por $\left(0,1,1,2,4,7,13,24,44, \ldots, \mathrm{k}_{n}, \ldots\right)$ e $\left(1,0,1,2,3,6,11,20,37, \ldots, 1_{n}, \ldots\right)$. Ao decorrer da atividade, os alunos devem ser instigados a descobrir ainda outras relações, como $\left\{l_{0}=k_{1}-k_{0}, \mathrm{n} \geq 2\right.$ e $\left.l_{n}=k_{n-1}+k_{n-2}\right\}$. No que concerne ao segundo questionamento, os alunos devem ser estimulados a perceber profundas ligações entre as sequências $\left\{p_{n}\right\}_{n \in Z}$ e $\left\{q_{n}\right\}_{n \in Z}$ com a sequência original de Fibonacci e, com um amparo em discussão pelo grupo, analisar a adequabilidade das terminologias que indicamos por "sequências extendidas tribonacci” e "sequências extendidas tetranacci”. Sugerimos, pois, a exploração aritmética de certas relações que já discutimos (ALVES; BORGES NETO, 2011), para o caso das sequências $\left\{p_{n}\right\}_{n \in Z}(\mathrm{I})$ e $\left\{q_{n}\right\}_{n \in Z}$ (II). Por exemplo, as relações $p_{n-3}=p_{n}-p_{n-1}-p_{n-2}$ e $\mathrm{q}_{n-4}=q_{n}-q_{n-1}-q_{n-2}-q_{n-3}$ devem ser exploradas, a fim de obter sua descrição no campo dos inteiros. Ademais, podemos obter: $p_{2}=p_{1}+p_{0}+p_{-1} \therefore p_{-1}=p_{2}-p_{1}-p_{0}$. Mas, se assumirmos que $p_{0}=0, p_{1}=p_{2}=1$, inferimos que $p_{-1}=0$. De modo similar, obteremos ainda que $p_{1}=p_{0}+p_{-1}+p_{-2} \therefore p_{-2}=p_{1}-p_{0}-p_{-1}=1 \mathrm{e}$ $p_{0}=p_{-1}+p_{-2}+p_{-3} \therefore p_{-3}=p_{0}-p_{-1}-p_{-2}=-1$. Ora, vale assinalar que Waddill e Sacks $(1967$, p. 215) sugerem, simplesmente, substituir ' $\mathrm{n}$ ' na expressão $p_{n}=p_{n-1}+p_{n-2}+p_{n-3}$ por ' $-n$ ', obtendo $p_{-n}=p_{-n+3}-p_{-n+2}-p_{-n+1}$, para $n>0$. Doravante, semelhantemente aos trabalhos de Alves e Borges Neto (2011), o grupo de estudantes, participantes da investigação, poderá apreciar a adequabilidade das nomenclaturas sugeridas ou, até mesmo, escolher outra.

Por outro lado, no $3^{\circ}$ questionamento, definindo a sequência sugerida $\left\{R_{\tilde{n}}\right\}_{n \in I N}$ de modo que $\left\{R_{0}=p_{1}-p_{0}\right.$ e $\left.R_{n}=p_{n-1}+p_{n-2}\right\}$, devemos estimular a identificação das seguintes relações algébricas: $R_{n}=\left(p_{n-1}+p_{n-2}\right)=\left(p_{n-2}+p_{n-3}+p_{n-4}\right)+\left(p_{n-3}+p_{n-4}+p_{n-5}\right)=$ $=\left(p_{n-2}+p_{n-3}\right)+\left(p_{n-3}+p_{n-4}\right)+\left(p_{n-4}+p_{n-5}\right)=R_{n-1}+R_{n-2}+R_{n-3} \therefore R_{n}=R_{n-1}+R_{n-2}+R_{n-3}$. Portanto, a sequência recursiva $\left\{R_{\tilde{n}}\right\}_{n \in I N}$, semelhantemente à sequência $\left\{p_{n}\right\}_{n \in I N}(\mathrm{I})$, é definida também a partir de três termos consecutivos. O elemento conceitual a ser discutido refere-se ao fato de que, a partir de uma sequência definida a partir do três termos consecutivos, formulamos uma outra que preserva as propriedades (recursividade a partir de três termos antecedentes) da sequência inicial. 
A fim de responder ao $4^{\circ}$ questionamento, de acordo com a definição, escrevemos $p_{n=1}=p_{n}+p_{n-1}+p_{n-2} \leftrightarrow\left(\frac{p_{n+1}}{p_{n-1}}\right)=\frac{p_{n}}{p_{n-1}}+1+\frac{p_{n-2}}{p_{n-1}}(*)$, e, em seguida, semelhantemente ao expediente usado em Feinberg (1963, p. $72-73)$, definimos $\frac{p_{n+1}}{p_{n}}=t_{n} ; \frac{p_{n}}{p_{n-1}}=t_{n-1} ; \frac{p_{n-1}}{p_{n-2}}=t_{n-2}$, tendo ainda que $\frac{p_{n+1}}{p_{n-1}}=\frac{p_{n+1}}{p_{n-1}} \cdot \frac{p_{n}}{p_{n}}=\frac{p_{n+1}}{p_{n}} \cdot \frac{p_{n}}{p_{n-1}}=t_{n} \cdot t_{n-1} . \quad$ Desse $\quad$ modo, estabelecemos: $t_{n} \cdot t_{n-1}=t_{n-1}+1+\frac{1}{t_{n-2}} \leftrightarrow t_{n}=1+\frac{1}{t_{n-1}}+\frac{1}{t_{n-1} \cdot t_{n-2}}$. Feinberg (1963, p. 73) admite a convergência da sequência há pouco definida por $\left\{t_{n}\right\}_{n \in I N}$, digamos, para um valor 'x'. Então, concluímos que $t_{n}=1+\frac{1}{t_{n-1}}+\frac{1}{t_{n-1} \cdot t_{n-2}} \rightarrow x=1+\frac{1}{x}+\frac{1}{x^{2}} \leftrightarrow x^{3}-x^{2}-x-1=0$. Koshy (2001, p. 240) recorda os valores numéricos das raízes da equação $x^{2}-x-1=0$, indicando-os por $\frac{1 \pm \sqrt{5}}{2} \in I R$. Com auxílio da figura 1, podemos estimular os estudantes a visualizar os valores correspondentes às raízes reais da equação $x^{3}-x^{2}-x-1=0$ e uma outra, descrita por $x^{4}-x^{3}-x^{2}-x-1=0$. Reparemos ainda que, no uso da descrição das raízes da cúbica, podemos escrever o número $\phi_{3}=\frac{(1+\sqrt[3]{19-3 \sqrt{33}}+\sqrt[3]{19+3 \sqrt{33}})}{3}=1.839286755214 \ldots$ que seria uma espécie ou tipo de número "tri-áureo" $\phi_{3}$ e, com isto, ensejar a formulação do número "tetra-áureo" $\phi_{4}$ e, assim sucessivamente, com o escopo de empregar uma terminologia que se aproxima da conhecida descrição e origem do número de ouro $\phi_{2}=1+\sqrt{5} / 2$.

Por outro lado, com arrimo na figura 1, podemos estimular o debate em sala de aula, no sentido de apreciar o comportamento dos outros valores semelhantes ao número de ouro, extraído do processo de divisão de um segmento em razão áurea. Na figura, divisamos uma única raiz real da equação $x^{3}-x^{2}-x-1=0$. Não obstante, com origem ainda no gráfico abaixo, quando nos atemos à equação $x^{4}-x^{3}-x^{2}-x-1=0$, percebemos a existência de duas raízes reais, uma delas positiva, que denotaremos por $\phi_{4}$.

Item (II): os valores particulares e preliminares da sequência $q_{n}=q_{n-1}+q_{n-2}+q_{n-3}+\mathrm{q}_{n-4}, n \geq 4$ podem ser indicados por $(0,0,1,1,2,4,8,15, \ldots)$. No que concerne aos termos com índices negativos, escrevemos $q_{3}=q_{2}+q_{1}+q_{0}+\mathrm{q}_{-1} \therefore \mathrm{q}_{-1}=q_{3}-q_{2}-q_{1}-q_{0}=1-1-0-0=0 . \quad$ Pelo mesmo motivo, 
determinamos $\mathrm{q}_{-2}=q_{2}-q_{1}-q_{0}-q_{-1}=1-0-0-0=1$ e seguimos um raciocínio semelhante para $n<0$.

\section{Figura 1 - Visualização dos valores correspondentes ao raciocínio empregado por Feinberg.}

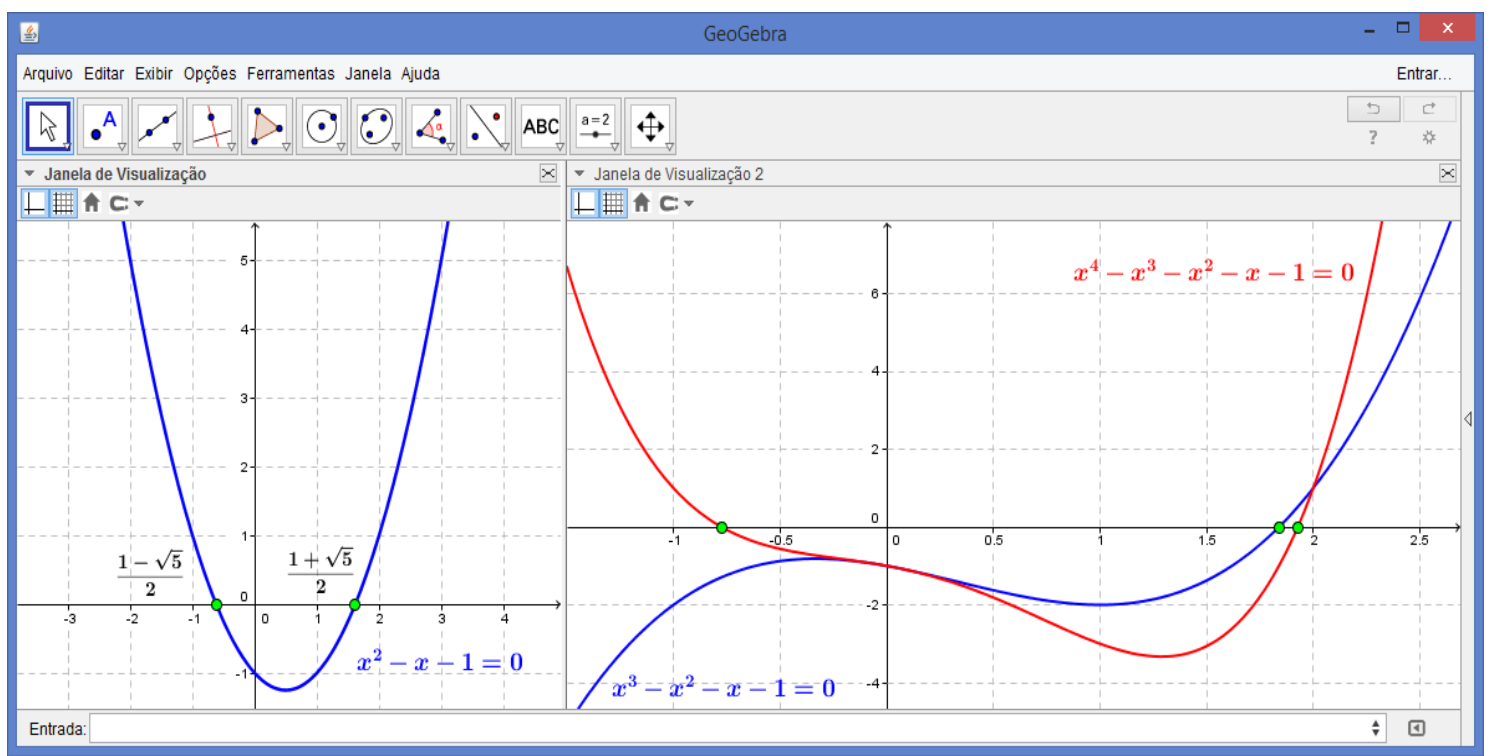

Fonte: Feinberg (1963).

Item (III): na expressão fornecida $(p, q, r, s, t, p+q+r+s+t, p+2 q+2 r+2 s+2 t, \ldots)$, os estudantes deverão reconhecer o padrão generalizante dos itens anteriores, à medida que, nas listas dos itens (I) e (II), podemos divisar uma relação de recorrência entre cada termo e os três (respectivamente, quatro) elementos antecedentes. Para testar alguns desses valores, podemos sugerir, por exemplo, que $p=q=r=s=t=1$ o que fornecerá: $(1,1,1,1,1,5,9,17,33,65,129,253, \ldots)$. Com arrimo no tirocínio mobilizado nos itens (I) e (II), o grupo deverá atingir o estabelecimento da formulação $r_{n}=r_{n-1}+r_{n-2}+r_{n-3}+r_{n-4}+r_{n-5}$, para $n \in Z$, que possui ainda características semelhantes à sequência Fibonacci, como a dependência de cinco termos antecessores e poderá proporcionar a descrição do número "penta-áureo" $\phi_{5}$, extraído de uma das raízes da equação $x^{5}-x^{4}-x^{3}-x^{2}-x-1=0$. Repetindo-se o argumento de Feinberg (1963, p. 72-73), proporcionamos a generalização dos argumentos descritos anteriormente.

\section{Considerações}

$\mathrm{Na}$ atividade proposta, observamos a ideia de proporcionar aos estudantes um raciocínio envolvendo o estabelecimento/descrição de definições, no contexto da investigação histórica envolvendo a noção SGF. Com efeito, de pouca divulgação na cultura acadêmica de graduação, outras noções de sequência nominadas por tribonacci, tetrabonacci, pentabonacci, hexbonacci, etc. (FENG, 2011; FEINBERG, 1963; MENDELSOHN， 1967; SARISAHIN; NALLI, 2014; 
WADDILL; SACKS, 1967), possibilitam um raciocínio generalizador, tomando como referência a emblemática sequência de Fibonacci $\left(f_{n}\right)_{n \in I N}$. Por outro lado, a atividade proposta visa instigar os alunos à ação de generalizar, formular descrições e nomenclaturas, nomes derivados do número de ouro " $\phi$ ", obter outros termos que indicaremos por $\left(\phi, \phi_{3}, \phi_{4}, \phi_{5}, \ldots, \phi_{n}, \ldots\right)$, bem como a elaborar definições formais (ALVES; BORGES NETO, 2011) para as propriedades descobertas no campo dos inteiros, num contexto de investigação histórica.

\section{Referências}

ALVES, F.R.V; BORGES NETO, H. A existência de sequência de Fibonacci no campo dos inteiros: uma atividade de investigação apoiada nos pressupostos da Sequência Fedathi. BOLETIM GEPEM. $n^{\circ}$ 59, 135 - 140, 2011. [online]

<http://www.ufrrj.br/SEER/index.php?journal=gepem\&page=article\&op=view\&path\%5B $\% 5 \mathrm{D}=64$ 7>. Acesso: 02/02/2015

ALVES, F.R.V. Uma discussão de artigos envolvendo propriedades da Sequência de Fibonacci apoiada na Tecnologia. Anais do VI HTEM. 1 - 17, 2013. [online]

<http://htem2013.dm.ufscar.br/anais/anais_do_vi_htem_ufscar_2013.html>.Acesso: 12/03/2015

FENG, J. More identities on the Fibonacci Numbers. Ars Combinatoria. $\mathbf{n}^{\mathbf{o}}$ 100, 73 - 78, 2011. [online]

<http://www.researchgate.net/profile/Jishe_Feng/publication/236343468_More_Identities_On_The _Tribonacci_Numbers/links/004635190c3a853293000000.pdf>. Acesso: 20/04/2015

FEINBERG, M. Fibonacci-Tribonacci. The Fibonacci Quartely. v. 1, no 3, October, 209 - 222. 1963. [online] < http://www.fq.math.ca/Scanned/1-3/feinberg.pdf>. Acesso: 02/02/2015

KOSHY, T. Fibonacci and Lucas Numbers with Applications. New York: John Wiley and Sons, 2011.

MENDELSOHN, P. N. The pentanacci numbers. The Fibonacci Quartely. v. 5, nº 3, October, 209 - 222. 1967. [online] < http://www.fq.math.ca/Books/Collection/mendelsohn.pdf> 2/02/15

SARISAHIN, T.; NALLI, A. On the pentanacci numbers. Mathematical and Computational Application. v. 19, $\mathrm{n}^{\circ} 3,255-262,2014$. [online]

<http://mcajournal.cbu.edu.tr/volume19/255.pdf>. Acesso: 12/04/2015.

SINGH, B.; BHADOURIA, P.; SIKHWAL, O.; SISODIYA, K. A Formula for Tetranacci-Like Sequence. General Mathematics Notes. v. 20, $\mathrm{n}^{\circ}$ 2, 136 - 141. 2014. [online]

<http://www.kurims.kyoto-u.ac.jp/EMIS/journals/GMN/yahoo_site_admin/assets/docs/10_GMN4642-V20N2.93181718.pdf> Acesso: 8/03/2015.

WADDILL, M. E; SACKS, L. Another Generalized Fibonacci Sequence. The Fibonacci Quartely. v. 5, no 3, October, 209 - 222. 1967. [online] 〈http://www.fq.math.ca/Scanned/5-3/waddill.pdf〉. Acesso: 12/05/2015. 